\title{
U n'esperienza didattica di espressione orale basata sul task approach
}

$M$ arco Presotto

Albert Vilagrasa

\begin{abstract}
This paper aims to present a language teaching/learning experience on the task-based approach, for Erasmus exchange students of I tal ian as FL.

This experiment acts as a stimulus towards language learning by providing the means whereby foreign language students may cover their most immediate contextual/cultural gaps. The paper presents the general steps followed, in order to confirm the potential of tasks with regard to modern language teaching/learning.
\end{abstract}

\section{Premessa}

Sono ampiamente note le riflessioni metodologiche che hanno portato a rivedere i limiti dell'approccio comunicativo; come si sa, pur avendo costituito un notevole passo in avanti nell'insegnamento di lingue straniere, questo approccio puo presentare il rischio di restringersi all'apprendimento di un insieme di contenuti che non implica necessariamente riuscire a comunicare; in altri termini, «sapere sulla comunicazione» non equivale a «saper comunicare».

Tralasciando l'ampia bibliografia che esiste sul tema, intendiamo qui come task approach nell'apprendimento di una lingua straniera un approccio basato su attività comunicative in cui viene coinvolto lo studente nella comprensione, manipolazione, produzione o interazione nella lingua straniera, e in cui la sua attenzione viene focalizzata principal mente sul significato piuttosto che sulla forma. Con un approccio per tasks si ha la possibilità di strutturare un corso perfettamente compatibile con un programma istituzionale in cui però non è il professore a stabilire e organizzare il contenuto del corso: sono infatti gli studenti a scegliere i centri d'interesse (o temi), e a partire da questi il professore fornisce i contenuti per dare strumenti e strategie allo scopo di risolvere necessità comunicative reali. In questo modo lo sviluppo della competenza comunicativa nella lingua straniera è concepito come lo sviluppo dei processi soggiacenti alla realizzazione delle attività reali di comunicazione. Le tasks costituiscono le unità sulle quali sviluppare il dominio di questi processi in maniera integrale a partire dalla competenza comunicativa nella L 1 che gli studenti posseggono. Come è noto, in un tale approccio ven- 
gono a costituirsi unità indivisibili di contenuto e metodologia, implicando la presa di decisione degli stessi studenti rispetto allo sviluppo e risoluzione delle tasks.

\section{II contesto}

L'insegnamento dell'italiano presso l'Escola d'I diomes M oderns dell'U niversitat de Barcelona si articola in cinque corsi ordinari (intensivi e estensivi) e un corso straordinario di mantenimento. Inoltre vengono impartiti corsi intensivi di 40 ore per studenti di diverse facoltà, ai quali I'U niversità ha aggiudicato borse di studio per un soggiorno in Italia di periodo variabile nell'ambito del progetto Erasmus/Socrates. $D$ ata la particolarità di questi studenti, abbiamo trovato necessario disegnare per loro un programma ad hoc allo scopo di coprire le immediate necessità di un approccio al contesto italiano partendo da una conoscenza pressocché nulla della lingua. 0 viamente, dal nostro punto di vista, adottare una impostazione per unità basandoci sui metodi comunicativi non ci avrebbe permesso di ottenere risultati tangibili.

Con il metodo adottato all'EIM, per i corsi ordinari, ci siamo trovati di fronte esattamente a queste limitazioni nel disegnare un corso specifico come quello citato, limitazioni ovviamente già apparse nei corsi ordinari, dove vengono modificate le unità con materiale supplementare vario. Ecco perché, data la specificità del corso di italiano per gli studenti Erasmus/Socrates, abbiamo creduto utile sperimentare in un tale contesto un tipo di corso nel quale i processi comunicativi reali in cui gli studenti si sarebbero visti coinvolti venissero forniti attraverso i contenuti comunicativi necessari per risolverli. Con queste premesse abbiamo deciso di sperimentare un corso basato sul task approach.

II primo ed evidente vantaggio di una tale prospettiva metodologica è quello dato dalla motivazione: gli studenti, investiti di un ruolo attivo nella strutturazione del corso, vedono a mano a mano risolti gli obiettivi comunicativi reali ai quali si troveranno di fronte in un futuro immediato (obiettivi che hanno scelto loro). Q uesto determina generalmente un potenziamento delle loro strategie comunicative ed una evidente crescita della loro sicurezza. U n secondo vantaggio altrettanto evidente è costituito dall'aumento di opportunità di pratica linguistica perché gli studenti vengono implicati in attività di risoluzione di problemi nelle quali devono negoziare il significato.

Infine, viene creata la possibilità di produrre un ventaglio più ampio di modelli linguistici, dato che è impossibile prevedere le soluzioni scelte o ricercate dallo studente.

\section{D escrizione della task}

D i seguito viene proposta la descrizione di una task che è stata sperimentata nel corso specifico per studenti Erasmus/Socrates nell'anno accademico 199495. Abbiamo scelto questa tra quelle che hanno costituito il corso perché ha 
avuto migliore accoglienza tra gli studenti nonché risultati particolarmente soddisfacenti.

A) N ella prima lezione abbiamo stimolato gli studenti a decidere tra di loro quali erano i centri di interesse - a partire da alcune proposte che abbiamo offerto, per rendere più agile la scelta ed evitare il rischio di divagare e allungare eccessivamente l'attività. In Tabelle 1 appaiono alcune delle proposte possibili a modo di esempio.

B) Abbiamo poi individuato i temi che hanno destato maggiore interesse e in questo modo abbiamo isolato le attività comunicative che gli studenti aspiravano a realizzare. A partire da queste informazioni sono stati analizzati gli obiettivi comunicativi implicati (cfr. Tabelle2).

C) Si è quindi proceduto alla programmazione delle tasks che avrebbero dimostrato il conseguimento degli obiettivi comunicativi, cioè quelle che avrebbero permesso agli studenti di dimostrare la capacità di «fare» qualcosa di concreto attraverso il linguaggio imparato. In Tabelle 3 appare l'esempio di una task, relativa al centro di interesse «ncontro», scelta tra quelle che hanno suscitato maggiore interesse e che ci servirà a partire da adesso come esempio.

Anche nelle fasi corrispondenti alle Tabelle 2 e Tabelle 3 è stata coinvolta la partecipazione degli studenti, perché è altamente positivo che vengano discussi tali obiettivi ai fini dell'influenza che avrà nella presa di coscienza del proprio apprendimento e per facilitare l'autovalutazione.

D) Per strutturare le tasks sono state specificate le componenti tematiche e linguistiche necessarie, e cioè contenuti nozionali e funzionali, grammaticali, e aree lessicali (cfr. Tabelle 4).

E) Si è proceduto alla pianificazione del processo; in questa fase, I'unità, articolata in forma di lezioni composte da tasks, è pianificata usando il materiale elaborato nelle fasi precedenti. In altri termini, ogni lezione prepara e alimenta la task finale. Cosi il processo che ci porterà al raggiungimento delle tasks finali consisterà in una serie di tasks minori distribuite in ogni lezione.

In Tabelle 5 viene offerta una parte del materiale che è stato preparato; tale materiale si riferisce solamente all'espressione orale, che è l'abilità a cui abbiamo dato maggiore rilevanza; si è comunque lavorato su tutte le abilità, ma data la peculiarità del corso abbiamo ritenuto più proficuo potenziare questa. Abbiamo diviso l'unità in cinque parti, ognuna delle quali aveva come finalità una task; il tempo dedicato a questa unità è stato calcolato in 10 ore.

Presentiamo qui alcune delle attività che sono state proposte all'interno di queste unità. In T abelle 6 appaiono come esempio le attività orali, riferite al punto 4: «Che cosa facciamo?», che abbiamo isolato per 
arrivare alla proposta di una task (inchiesta). Si tratta qui della struttura portante della lezione, che deve comunque essere integrata da altri materiali in cui vengano implicate tutte le abilità.

F) Un ultimo aspetto che è stato contemplato riguarda la valutazione. Se un'unità viene strutturata intorno a delle tasks, cioè intorno ad obiettivi concreti, è perfettamente realizzabile una valutazione riferita a ciascuno degli obiettivi che dovrebbero essere stati raggiunti. Come appare evidente, in un approccio di questo tipo lo studente viene coinvolto in modo attivo in tutte le fasi di realizzazione del corso e quindi risulta particolarmente importante che esista una forma di autovalutazione.

Abbiamo elaborato delle schede di autovalutazione per gli studenti per ognuna delle unità. In Tabelle 7 proponiamo un possibile esempio relativo alla task che abbiamo descritto finora.

\section{Conclusione}

L'esperienza didattica che abbiamo delineato è il frutto di una nostra riflessione metodologica sull'insegnamento dell'italiano come lingua straniera e nel caso particolare di corsi specifici come quello che abbiamo visto; non pretendiamo certo di aver offerto soluzioni, ma semplicemente di aver descritto un esempio di applicazione dell'approccio per tasks che per noi rappresenta un proposta didattica valida e altamente proficua. La grande flessibilità e le molteplici possibilità di applicazione ai diversi contesti reali di insegnamento rende questo approccio un interessante strumento pedagogico per ampliare e migliorare il lavoro del professore.

\section{Appendice: T abelle descrittive}

T abelle 1. Centri di interesse.

- nuovi amici

- rapporti con i professori

- I'appartamento che desideriamo

- un incontro (una cena)

- la nostra città

- abitudini alimentari

- la famiglia

- una gita

- ... / ... 
T abelle 2. 0 biettivi comunicativi [C entro di interesse: «un incontro»].

- chiedere e dire l'ora.

- chiedere informazioni e informare sugli orari.

- chiedere informazioni e informare sulla localizzazione nello spazio.

- esprimere gusti, preferenze e opinioni sulle attività del tempo libero.

- chiedere e informare sulle disponibilità orarie.

- esprimere accordo e disaccordo.

$-\ldots / \ldots$

Tabelle 3. T ask finale

Prendere appuntamento con qualcuno (IL gruppo)

PER USCIRE IN SIEME: DECIDERE L'ATTIVITÀ, IL GIORNO, L'ORA E IL LUOGO.

\section{Tabelle 4.}

\begin{tabular}{|c|c|c|c|}
\hline \multirow[b]{2}{*}{$\begin{array}{l}\text { Componenti } \\
\text { tematiche }\end{array}$} & \multicolumn{3}{|c|}{ Componenti linguistiche } \\
\hline & $\begin{array}{l}\text { Contenuti nozionali } \\
\text { e funzionali }\end{array}$ & $\begin{array}{c}\text { Contenuti } \\
\text { grammaticali }\end{array}$ & Contenuti lessicali \\
\hline $\begin{array}{l}\text { - tempo } \\
\text {-localizzazione } \\
\text {-opinioni, } \\
\text { preferenze } \\
\text {...... }\end{array}$ & $\begin{array}{l}\text { - chiedere e dire } \\
\text { l'ora } \\
\text { — chiedere } \\
\text { informazioni e } \\
\text { informare sugli } \\
\text { orari degli } \\
\text { spettacoli, negozi, } \\
\text { trasporti pubblici } \\
\text { e altri servizi } \\
\text { — chiedere } \\
\text { informazioni e } \\
\text { informare sulla } \\
\text { localizzazione } \\
\text { nello spazio } \\
\text { - esprimere gusti, } \\
\text { preferenze e } \\
\text { opinioni sulle } \\
\text { attività del tempo } \\
\text { libero } \\
\text {-chiedere e } \\
\text { informare sulle } \\
\text { disponibilità orarie } \\
\text { —esprimere accordo } \\
\text { e disaccordo }\end{array}$ & $\begin{array}{l}\text { - agg. interrogativo } \\
\text { «che» } \\
\text { — agg. indef. «ogni», } \\
\text { «qualche», } \\
\text { «alcuni» ecc. } \\
\text { — preposizioni con } \\
\text { significato } \\
\text { temporale, «da», } \\
\text { «fino a», «a», } \\
\text { «verso» } \\
\text { —avverbi e } \\
\text { locuzioni } \\
\text { temporali, } \\
\text { «domani», } \\
\text { «a volte» } \\
\text { - verbi: presente } \\
\text { indicativo, } \\
\text { «essere», } \\
\text { «arrivare», } \\
\text { «uscire», «partire», } \\
\text { «iniziare», } \\
\text { «finire», «aprire», } \\
\text { «chiudere» } \\
\text {../... }\end{array}$ & $\begin{array}{l}\text { - lessico per } \\
\text { misurare il tempo } \\
\text { — nomi di spettacoli, } \\
\text { negozi, servizi } \\
\text { pubblici, trasporti } \\
\text { — parti del giorno } \\
\text { — giorno della } \\
\text { settimana } \\
\text { _alcuni avverbi di } \\
\text { frequenza }\end{array}$ \\
\hline
\end{tabular}




\section{T abelle 5.}

\section{Pianificazione del PROCESSO: L'UNITÀ}

TASK FIN ALE: PRENDERE APPUNTAMENTO CON QUALCUNO (IL GRUPPO) PER USCIRE IN SIEME; DECIDERE L'ATTIVITÀ, IL GIORNO, L'ORA E IL LUOGO.
1) Che ora è?
2) A che ora...
3) D ov'è?
4) Che cosa facciamo?
5) Come rimaniamo?

T abelle 6.

\section{CHE COSA FACCIAM O?}

1. D iscuti con un tuo compagno se ti piace o non ti piace:

- Ti piace... ?

- No, non mi piace... / Sì, mi piace...

andare a sciare / prendere il sole / andare al cinema / andare a teatro / giocare a carte / uscire di sera / dormire fino a tardi / alzarti presto i giorni di festa / fare viaggi organizzati / ... / ...

- Ti piace/ piacciono ... ?

- No, non mi piace / piacciono ...

i film del terrore / i ristoranti cinesi / lo zampone / le partite di calcio / gli spaghetti / ... $/ \ldots$

2. Chiedi ai tuoi compagni di lezione qual è il colore che gli piace di più e formate dei gruppi secondo le preferenze:

- Qual èil colorecheti piace di più?

- II giallo

3. M ettiti d'accordo con i compagni del tuo gruppo su quale giorno della settimana vi piace di più:

- Io preferisco il venerdì / M i piace di più il venerdì

\section{4. $\mathrm{N}$ el gruppo che avete formato ordinate a seconda delle vostre preferenze:}

registi di cinema / attori di cinema / attrici di cinema / cantanti / piatti gastronomici / bibite / ... / ... 


\section{T rova qualcuno nell'aula che abbia i tuoi stessi gusti:}

Q ual èil Paese d'Europa cheti piace di più?/ Preferisci andareal mareo in montagna? / Ti piace di più leggere o guardare la TV?/

6. D isegna una inchiesta con risposta multiple sugli interessi dei tuoi compagni di lezione (o di altri gruppi). Proponila e fai una breve relazione sui risultati:

- Che cosa preferisci fareil sabato sera?
a) andare in discoteca
b) rimanere a casa
c) andare al cinema
d) giocare a carte

\section{T abelle 7.}

\section{Autovalutazione}

TASK FIN ALE : Prendere APPuntamento CON QUALCUNo (IL GRUPPO) PER USCIRE IN SIEME; DECIDERE L'ATTIVITÀ, IL GIORNO, L'ORA E IL LUOGO.

Sei in grado di fare queste cose? Segnalo con una croce. Sono in grado di ?

$\mathbf{1}=\mathrm{N}$, per niente

$\mathbf{2}=\mathrm{N}$ o, non molto

$\mathbf{3}=$ Cos̀̀ cos̀̀

4 = Sì, abbastanza

5 = Sì, benissimo

1) chiedere l'ora

2) dire l'ora

3) capire le informazioni orarie

4) chiedere informazioni sugli orari

5) dare informazioni sugli orari

6) capire le informazioni sugli orari

7) esprimere gusti e preferenze

8) chiedere gusti e preferenze

9) ... $/ \ldots$ 


\section{Bibliografia}

AA.VV. (1990). «D ossier enfoque por tareas». Cable (Revista didáctica del E/LE), V.

CAN DLIN, C.N .; M URPHY, D. (1987) (a cura di). Lancaster Practical Papersin English Language Education: Vol. 7. Language Learning Tasks. Englewood Cliffs. N ew Jersey: Prentice H all.

EstalRe, S.; ZANóN, J. (1990). «El diseño de unidades didácticas en L2 mediante tareas: principios y desarrollo». Comunicación, lenguaje y educación, 7-8: 55-90.

- (1993). Planning Classwork: a T ask Based Approach. O xford: H einemann.

Long, M.H.; CROokes, G. (1992). «Three Approaches to Task-Based Syllabus D esign». T esol Q uarterly, XXVI: 27-56.

N unam, D. (1989). Designing Task for the Communicative Classroom. Cambridge U niversity Press. 